\title{
Impaired performance in a working memory binding task in patients with schizophrenia
}

\author{
Franck Burglen ${ }^{\mathrm{a}}$, Philippe Marczewski ${ }^{\mathrm{b}}$, Karen J. Mitchell ${ }^{\mathrm{c}}$, Martial van der Linden ${ }^{\mathrm{d}}$, \\ Marcia K. Johnson ${ }^{c}$, Jean-Marie Daniona, Pierre Salaméa,* \\ a INSERM U. 405, Psychopathologie et Pharmacologie de la Cognition, Department of Psychiatry, Hôpital Civil, \\ 67091 Strasbourg Cedex, France \\ ${ }^{\mathrm{b}}$ Liège University, Liège, Belgium \\ ${ }^{\mathrm{c}}$ Department of Psychology, Yale University, New Haven, CT, USA \\ ${ }^{\mathrm{d}}$ Geneva University, Geneva, Switzerland
}

Received 25 February 2003; received in revised form 5 November 2003; accepted 18 December 2003

\begin{abstract}
This study investigated feature binding in a working memory task in patients with schizophrenia and in normal controls. Twenty-five patients and 25 controls participated. On each trial, three drawings of familiar objects were presented sequentially, each in a different cell of a $3 \times 3$ grid. In different blocks of trials, participants remembered either individual features (object and location conditions) or an object and its location (combination condition). The results showed that patients were slower and less accurate than controls under all conditions. Accuracy of both groups was reduced in the combination condition relative to the single-feature conditions, but patients showed disproportionately poorer performance in the combination condition than in the object and location conditions. Thus, patients with schizophrenia exhibit deficits in working memory, particularly when the task requires binding objects to their locations. This finding demonstrates that processes that establish coherent and temporary episodic representations in working memory are impaired in schizophrenia.
\end{abstract}

(C) 2003 Elsevier Ireland Ltd. All rights reserved.

Keywords: Schizophrenia; Working memory; Object; Location; Associations; Memory binding

\section{Introduction}

An episodic memory is comprised of a collection of different features that are associated to form a coherent representation of an event. Features include, for example, the physical properties of the event and the time when or place where the

\footnotetext{
*Corresponding author. Tel.: +33-3-88-11-64-62; fax: + 33-3-88-11-64-46.

E-mail address: salame@alsace.u-strasbg.fr (P. Salamé).
}

event occurred, i.e. in addition to the ability to retain the features themselves, a key process in episodic memory is the ability to form associations between the features experienced together, a process called 'memory binding' (e.g. Johnson and Chalfonte, 1994). Schizophrenia is associated with episodic memory deficits (for reviews, see Heinrichs and Zakzanis, 1998; Aleman et al., 1999). For example, patients with schizophrenia exhibit impairments of memory for temporal information 
(Schwartz et al., 1991; Rizzo et al., 1996), frequency of occurrence of events (Gras-Vincendon et al., 1994), and reality monitoring (i.e. determining if information was self-generated or provided by the experimenter) (Bentall et al., 1991; Rankin and O'Carroll, 1995). Such findings raise the possibility that episodic memory deficits in patients with schizophrenia may be related to a failure of binding features together to create more complex representations (Rizzo et al., 1996; Danion et al., 1999).

Most studies, however, do not separately assess memory for individual features and memory for bound features. An exception is a study by Rizzo et al. (1996). They presented schizophrenic patients and healthy controls with a grid in which 30 words were displayed successively. They asked the participants to study the words, and no mention was made of locations. Following a 5-min delay, during which unrelated tasks were carried out, participants received a memory test in which they were asked (a) to distinguish a word they had seen previously from two lures consisting of items that had not been presented earlier; (b) to decide which of three locations (two of which had not been previously occupied) was linked with a specific word; and (c) to decide which of three studied words had previously been displayed in a specific location. Whereas the first and second tasks could be performed simply by eliminating those items or locations that had not been used, successful performance in the third (combination) condition relied on the ability to create a link between the word and its precise location (i.e. to bind word and location together). Patients with schizophrenia exhibited a disproportionate deficit in the combination condition, suggesting dysfunction in their ability to bind words and spatial information.

Memory binding deficits in this long-term memory task could equally result from impaired processing at encoding, during retention, or at test. However, because there is consistent evidence that patients with schizophrenia fail to organize and elaborate ongoing experience, Rizzo et al. (1996) proposed that the origin of the binding deficit may be at the early stage of encoding that comprises the critical processes for establishing associations between the different features of events, that is, in working memory.

Working memory (WM) is usually thought of as a number of limited capacity and specialized systems devoted to temporary storage and on-line manipulation of information (Baddeley, 1986), and most studies of WM have assessed memory for specific types of information (i.e. features). For example, there is considerable evidence for a phonological loop and visuo-spatial sketch pad that are responsible for the temporary storage and maintenance of speech-based and visuo-spatial material, respectively (Baddeley, 2000a). More recently, investigators have begun to explore WM for combinations of features such as objects and their locations (Mitchell et al., 2000a,b; Prabhakaran et al., 2000). This new emphasis on feature binding in WM reflects a growing appreciation of the centrality of WM to cognition, which has recently led Baddeley (2000b) to add a new hypothetical component, the episodic buffer, to the initial tripartite WM model. The episodic buffer is proposed to be a storage space for temporary representations (presumably feature combinations) being entered into or retrieved from episodic longterm memory. Although several studies demonstrate impaired performance of patients with schizophrenia in tasks that tap visuo-spatial and executive components of WM (Salamé et al., 1998; Keefe, 2000; Glahn et al., 2003), very little experimental work has been devoted to a direct investigation of the process of memory binding in $\mathrm{WM}$ and the construction of temporary episodes in on-line processing.

The present study explores the ability of schizophrenic patients and normal controls to establish temporary links between pairs of objects and locations in a WM setting, using a paradigm initially introduced by Mitchell et al. (2000b). The task was comparable to that of Rizzo et al. (1996) with two exceptions. Firstly, binding was intentional, not incidental (i.e. participants received explicit instructions to remember either object, location, or object and location information on each trial). Secondly, a trial included only three items presented for durations that were comparable to those generally adopted in short-term memory span tasks or in serial recall, i.e. $1000 \mathrm{~ms}$ per 
item, unlike the substantially longer durations used in most long-term memory paradigms. Also, the delay was unfilled and lasted $8 \mathrm{~s}$, thus permitting some form of rehearsal of the stimulus, unlike long-term memory paradigms in which the delay is typically on the order of minutes or even longer, and thus typically includes interpolated activities, as in Rizzo et al. (1996).

Participants were presented with successive blocks of trials in which they were explicitly asked to remember only the objects, only the locations occupied in a grid (single-feature conditions), or the objects and their associated locations in the grid (combination condition). They were then tested on each trial with a single yes/no recognition probe following an 8-s unfilled delay. Consistent with the evidence that patients with schizophrenia show visuo-spatial and executive disruptions, it was expected that their performance should be particularly reduced in the combination condition, as compared with the single-feature conditions.

\section{Methods}

\subsection{Participants}

Fifty participants were in the study, 25 patients and 25 healthy controls. The patients were seven women and 18 men (mean age $=37.64$; S.D. $=$ 7.53 years; mean educational level $=12.64$; S.D. $=$ 3.04 years); all were outpatients who met DSMIV (American Psychiatric Association, 1994) criteria for schizophrenia (paranoid, $N=23$; residual, $N=1$; undifferentiated, $N=1$ ) as determined by consensus of the current treating psychiatrist and two senior psychiatrists belonging to the research team. No participants had a history of traumatic brain injury, epilepsy, substance abuse, other diagnosable neurological conditions or organic mental disorders, nor were they being treated with antidepressants, benzodiazepines or lithium. All patients were clinically stabilized at the time of test. Twelve patients were receiving atypical neuroleptics (clozapine, risperidone, amisulpride or olanzapine) and 13 patients were receiving typical neuroleptics (mean equivalent dose $=187$; S.D. $=$ $83 \mathrm{mg}$ of chlorpromazine). In addition to neuro- leptic treatment, nine patients were also receiving an anti-parkinsonian treatment (trihexiphenidyle, tropatepine). Mean scores of the Brief Psychiatric Rating Scale, the Scale for the Assessment of Positive Symptoms and the Scale for the Assessment of Negative Symptoms were 45.80 (S.D. = 12.66), 28.52 (S.D. = 19.56) and 28.20 (S.D. $=$ 19.84), respectively.

Twenty-five healthy control participants -12 women and 13 men-were also recruited (mean age $=36.16$, S.D. $=4.67$ years; mean educational level $=12.92$, S.D. $=2.31$ years). None had a history of alcoholism, drug abuse, neurological or psychiatric illness, and none were taking any drugs. The two groups of participants did not differ in age, educational level or IQ, as measured using the Wechsler Adult Intelligence ScaleRevised (Wechsler, 1981) (patients: mean $=97.28$, S.D. $=15.37$; controls: mean $=97.60$, S.D. $=8.60)$, $\left(t_{48}<1\right.$ in each case). The local Ethical Committee approved the study, and each participant signed an informed consent form before the experiment and received a payment for taking part.

\subsection{The WM task}

The WM task assessed participants' ability to remember drawings of common objects or their locations in a nine-cell grid, or objects together with their locations (see Mitchell et al., 2000b, for a figure depicting the task). Each trial started with the condition name ('object', 'location' or 'object+location') displayed in the center of a Macintosh computer color screen (model LC 475) for $500 \mathrm{~ms}$, reminding participants what they were to remember on that trial. Then three successive monochromatic drawings (Snodgrass and Vanderwart, 1980) of familiar objects (e.g. trumpet, bell, airplane) appeared in a $3 \times 3$ grid, each occupying a different cell, or location. Each grid cell was approximately $7 \mathrm{~cm}^{2}$. For generality, two sets of eight different object drawings were used (for any one participant, the untested set was used for practice trials). The presentation of each stimulus lasted $1000 \mathrm{~ms}$, and all locations except the center cell were used for stimulus presentation. Colors of the three objects and their locations in the grid cells in a trial were always different. Participants 
were explicitly told beforehand that the object's color was irrelevant, i.e. they would not be tested for color. Following the presentation of the third stimulus, an unfilled delay of $8000 \mathrm{~ms}$ was allowed for rehearsal during which the screen was blank. The delay ended with the presentation of the word 'Test' for $500 \mathrm{~ms}$ followed by a probe. In the object condition, the probe consisted of a blackand-white drawing presented in the center cell of the grid, and the participant had to decide whether the probe corresponded to one of the objects presented on that trial. In the location condition, the probe was a large black dot presented in any cell of the grid except the center one and the participant had to decide whether the cell had been occupied by an object on that trial. In the combination condition, the probe consisted of a blackand-white drawing of an object that was presented in one cell of the grid, except for the center one, and the participant had to decide if that object was presented in its correct location. A probe was presented for $2000 \mathrm{~ms}$, but the total response period lasted $4000 \mathrm{~ms}$ during which participants had to decide whether a test item was a target or lure (described below) and respond using the two specified buttons of the computer keyboard. Righthanded participants used their index and middle fingers to press the J and I keys for 'yes' and 'no' responses, respectively, while left-handed participants pressed the ' $\mathrm{W}$ ' and ' $\mathrm{D}$ ' keys. Participants were instructed to respond as quickly as possible without sacrificing accuracy. Psyscope software (Cohen et al., 1993) was used to present the stimuli and collect data (response and response time $[\mathrm{ms}]$ ).

Each condition comprised an equal number of targets and lures in random order. A target corresponded to an object, or location, or object+ location that had been presented in the trial (i.e. a 'studied' item from that trial) and they were taken equally often from each ordinal study position. For the object and location conditions, a lure corresponded to an item that was not presented on the current trial, though it would have been presented on previous trials because the objects and locations were repeated across trials. In the combination condition, a lure corresponded to an object presented in the same trial but located in a cell of the grid occupied by another object in that trial, i.e. lures consisted of presented but re-paired objects and locations.

\subsection{Procedure}

The practice session started with a detailed description of the task and the instructions associated with each condition. This was followed by an interactive demonstration using three different tokens and a drawing of a $3 \times 3$ grid that aimed at providing additional information about the content of the task and to check the participant's understanding of the whole procedure. Then the computerized version of the task was practiced first in the object condition that comprised two target trials alternating with two lure trials. After a short pause, this procedure was repeated for the location and then for the combination condition. All participants practiced the three conditions in this order, and the practice trials of each condition were the same for all participants. This procedure ensured a full understanding of the task requirements and allowed each participant to reach a level of perfect performance at training. The experimental session consisted of 108 trials arranged in three blocks of 36 trials (12 trials per condition). Two different orders of conditions were counterbalanced across participants in each group. Half of the participants started with the object condition and the other with location. Thus, in neither order did the participants start the experiment with the combination condition.

\subsection{Data analysis}

Test data were analyzed using BMDP 7.0 statistical software. Comparisons involved analyses of variance (ANOVA) on accuracy scores and response times. Analyses included group (controls vs. patients) as between-subject factor, and condition (object, location, combination) and test item type (target vs. lure) when needed, as withinsubject factors. Student's $t$-tests and NewmanKeul's $(\mathrm{N}-\mathrm{K})$ tests for multiple comparisons were used for post hoc analyses. In all comparisons, the alpha level was set at 0.05 . Greenhouse-Geisser's 
probability corrections were applied for multiple comparisons.

\section{Results}

\subsection{Accuracy}

The mean proportions of hits $(\mathrm{H})$, false alarms (FA), and corrected recognition ( $\mathrm{H}$ minus FA) for each condition of each group are given in the first two rows of Table 1 .

An analysis of corrected recognition scores showed an overall effect of condition $\left(F_{2,96}=\right.$ $13.15, P<0.01)$; subsequent $\mathrm{N}-\mathrm{K}$ tests showed that the object and location conditions did not differ from each other $(P>0.05)$, but each differed significantly $(P<0.05)$ from the combination condition. There was an effect of group $\left(F_{1,48}=11.38\right.$, $P<0.01$ ), with patients' scores being lower than controls' (0.78 vs. 0.90 , respectively). There was also a significant condition $\times$ group interaction $\left(F_{2,96}=4.45, P<0.03\right)$, reflecting a disproportionate decrease in patient's performance in the combination condition. As can be seen in Table 1, this interaction was primarily a consequence of the high false alarm rate of patients in the combination condition.

Secondary analyses were performed to investigate whether patients' continued to show a decrement in performance on the combination trials under conditions where the performance of patients and controls was the same in the single-feature trials. The data of six patients whose performance in the single-feature trials was more than 4 S.D. lower than that of controls were excluded. The

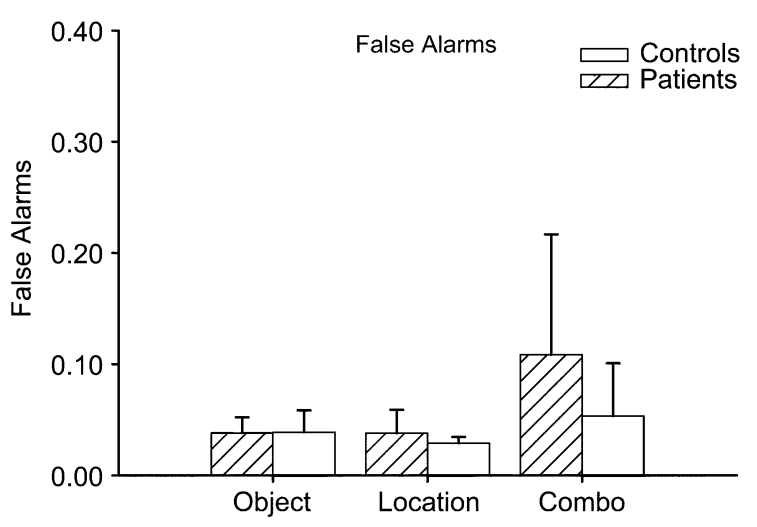

Fig. 1. Mean (S.D.) proportion of false alarms as a function of condition in 19 patients with schizophrenia (see text) and 25 healthy participants.

mean hit, FA and corrected recognition scores of the remaining 19 patients are shown in the last row of Table 1 .

An ANOVA comparing the corrected recognition of these 19 patients to the 25 controls showed an effect of condition $\left(F_{2,84}=9.50, P<0.01\right)$, but no effect of group $\left(F_{1,42}=3.25, P>0.05\right)$, and the group $\times$ condition interaction was not significant $\left(F_{2,84}=2.02, P>0.05\right)$. Analysis of FA, however, showed an effect of group $\left(F_{1,42}=5.70, P<0.03\right)$, an effect of condition $\left(F_{2,84}=13.76, P<0.01\right)$ and a significant group $\times$ condition interaction $\left(F_{2,84}=\right.$ 4.47, $P<0.04)$. As shown in Fig. 1, patients whose performance was intact in the single-feature conditions still exhibited significant difficulty rejecting lures in the combination condition.

Because our participants' samples comprised more males than females, particularly in the

Table 1

Mean (and S.D.) proportion of hits (H), false alarms (FA) and corrected recognition ( $\mathrm{CR}=\mathrm{H}-\mathrm{FA})$ as a function of condition for the full sample of 25 patients with schizophrenia and 25 healthy participants and a subset of 19 patients (see text)

\begin{tabular}{|c|c|c|c|c|c|c|c|c|c|}
\hline & \multicolumn{3}{|l|}{ Hits } & \multicolumn{3}{|c|}{ False alarms } & \multicolumn{3}{|c|}{ Corrected recognition } \\
\hline & Object & Location & Combination & Object & Location & Combination & Object & Location & Combination \\
\hline $\begin{array}{l}\text { Patients } \\
\qquad(n=25)\end{array}$ & $\begin{array}{l}0.88 \\
(0.12)\end{array}$ & $\begin{array}{l}0.90 \\
(0.11)\end{array}$ & $\begin{array}{l}0.85 \\
(0.16)\end{array}$ & $\begin{array}{l}0.07 \\
(0.07)\end{array}$ & $\begin{array}{l}0.06 \\
(0.07)\end{array}$ & $\begin{array}{l}0.16 \\
(0.17)\end{array}$ & $\begin{array}{l}0.81 \\
(0.15)\end{array}$ & $\begin{array}{l}0.84 \\
(0.14)\end{array}$ & $\begin{array}{l}0.69 \\
(0.29)\end{array}$ \\
\hline $\begin{array}{l}\text { Controls } \\
\quad(n=25)\end{array}$ & $\begin{array}{l}0.95 \\
(0.05)\end{array}$ & $\begin{array}{l}0.95 \\
(0.03)\end{array}$ & $\begin{array}{l}0.93 \\
(0.06)\end{array}$ & $\begin{array}{l}0.04 \\
(0.02)\end{array}$ & $\begin{array}{l}0.03 \\
(0.01)\end{array}$ & $\begin{array}{l}0.05 \\
(0.05)\end{array}$ & $\begin{array}{l}0.91 \\
(0.05)\end{array}$ & $\begin{array}{l}0.92 \\
(0.03)\end{array}$ & $\begin{array}{l}0.88 \\
(0.07)\end{array}$ \\
\hline $\begin{array}{l}\text { Patients } \\
\quad(n=19)\end{array}$ & $\begin{array}{l}0.93 \\
(0.07)\end{array}$ & $\begin{array}{l}0.96 \\
(0.04)\end{array}$ & $\begin{array}{l}0.91 \\
(0.11)\end{array}$ & $\begin{array}{l}0.04 \\
(0.01)\end{array}$ & $\begin{array}{l}0.04 \\
(0.02)\end{array}$ & $\begin{array}{l}0.11 \\
(0.11)\end{array}$ & $\begin{array}{l}0.87 \\
(0.10)\end{array}$ & $\begin{array}{l}0.91 \\
(0.05)\end{array}$ & $\begin{array}{l}0.80 \\
(0.22)\end{array}$ \\
\hline
\end{tabular}




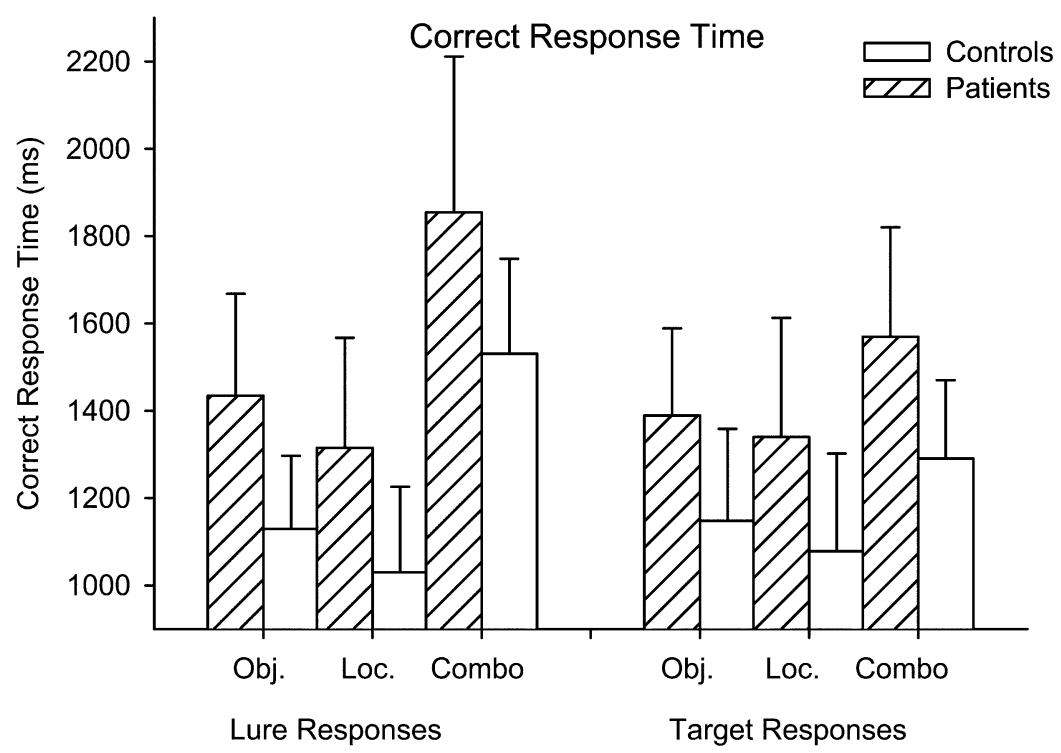

Fig. 2. Mean (S.D.) of correct response time in $\mathrm{ms}$ for lure and target responses as a function of condition in 25 patients with schizophrenia and 25 healthy participants.

patient's group, the last analysis considered the issue of whether gender may have influenced performance and included gender as a covariate. After adjustment by the gender factor, which was itself not significant $\left(F_{1,41}=3.13, P>0.05\right)$, the results still showed a significant difference between groups $\left(F_{1,41}=4.13, P<0.05\right)$ indicating that group differences in false alarms were not affected by gender. (Results of the condition factor and the group $\times$ condition interaction are not reported because they are unaffected by the between factor of gender.)

\subsection{Response time}

Mean correct response times as a function of group, condition, and the type of response (target, lure) are plotted in Fig. 2. Examination of this figure allows three main observations: (1) Response times were longer in the combination condition for both groups; (2) patients were slower than controls, regardless of condition and type of response; and (3) response times were longer for lures than for targets in the combination condition for both groups. These observations were all confirmed by the ANOVAs.
The analysis showed an overall effect of condition $\left(F_{2,96}=192.78, P<0.01\right)$, and a subsequent $\mathrm{N}-\mathrm{K}$ test revealed that the three conditions differed significantly from each other $(P<0.05)$, with location leading to the shortest response time, and combination of the longest. It also revealed a significant effect of group $\left(F_{1,48}=25.31, P<0.01\right)$, with patients being much slower than controls. There was an effect of test item type $\left(F_{1,48}=\right.$ 19.05, $P<0.01)$ reflecting longer response times for lures than targets, and finally, the analysis revealed a significant condition $\times$ item type interaction $\left(F_{2,96}=46.12, P<0.01\right)$. Subsequent analyses showed that mean response time for lures and targets did not differ in the object or location conditions $\left(F_{1,98}<1\right.$ in each case $)$, whereas in the combination condition, response time for lures was longer than that of targets $\left(F_{1,98}=19.34, P<0.01\right)$. In other words, all participants took longer to reject lures correctly when feature binding was required. None of the other interactions were significant.

\subsection{Additional analyses}

Since all patients were receiving neuroleptic medication, a number of additional analyses 
attempted to examine the question of whether drug treatment may have biased the finding of WM impairment. To this end, a number of variables including age, education, IQ, correct response time to lures and targets, and FA in each condition were compared in the two subgroups of patients defined by their typical or atypical drug intake, using successive Student's $t$-tests. None of the analyses showed any significant difference between the two subgroups, indicating that the overall level of patients' performance was not likely to be influenced by their current neuroleptic medication.

Another set of analyses examined the potential correlations between BPRS, SAPS and SANS symptom ratings and speed and accuracy indexes. The results showed that BPRS and SAPS correlated negatively with $\mathrm{H}$ in the combination condition $(r=-0.391, \quad P<0.05 ; \quad r=-0.462, \quad P<0.02$; respectively). SAPS ratings also correlated negatively with H-FA in combination $(r=-0.415, P<$ $0.04)$. Finally, SANS correlated positively with mean correct response time in object and location conditions only $\left(r_{\mathrm{s}}=0.412\right.$ and 0.408 , d.f. $=23$, $P<0.04$ in both cases). Because these results failed to show any significant correlation with the crucial index of FA, the actual implications of the correlations observed would remain marginal.

\section{Discussion}

The study yielded three main results. First, performance was poorer when the participants were required to bind objects with locations than when they had to remember only the location or object. Second, an overall impairment of performance was observed in patients with schizophrenia, adding to the accumulating evidence of WM deficits in these patients. The impairment likely does not reflect lower general intellectual capacities of patients, because their IQ was matched with that of controls. The third, and new, finding was a disproportionate deficit exhibited by patients in the combination condition; relative to object and location, corrected recognition scores in the combination condition were disproportionately lower in patients than controls. Evidence that patients experienced particular difficulties in the combination condition was further supported by secondary anal- yses, which indicated that a subset of patients whose performance in the single-feature conditions did not differ from that of controls still produced higher levels of combination false alarms than controls. Note that in this procedure, combination condition performance on lures is most informative about memory for the unique association between the features studied on that trial because only by remembering how the features were originally combined can lures be rejected. Thus, the present findings extend to WM the observation that patients with schizophrenia experience difficulties binding content and spatial information in LTM (Rizzo et al., 1996). They are, however, at variance with those of Gold et al. (2003) who examined the retention of visual color and orientation features alone or in conjunction using a paradigm based on that of Luck and Vogel (1997). Their results showed similar rates of correct detections in patients with schizophrenia and controls, leading to the suggestion of an intact ability to bind features in WM in these patients. However, as recently pointed out by Wheeler and Treisman (2002), the assumption of an involvement of binding processes in Luck and Vogel's paradigm might actually be questioned on the ground that the two features (i.e. color and orientation) were always presented simultaneously bound, whichever the precise condition of test.

How can the pattern of performance exhibited by patients with schizophrenia in the WM binding task be explained? One explanation is related to the differential levels of difficulty of the experimental conditions. Overall, performance in accuracy and in speed of responses was lower in the combination condition than in the single-feature conditions, suggesting that the former was more difficult than the latter ones. Therefore, it could be argued that the decrease of patients' performance might simply reflect a general deficit of the WM processes involved in the task, which is more likely to show up as task difficulty increases (Chapman and Chapman, 1978). Another possibility is that the combination trials involved more information than the single-feature trials, and patients simply have difficulty maintaining this higher 'load' in WM. However, the load is only higher in the combination than the single feature 
trials if the items and locations have not been bound. Thus, we think that it is more likely that linking together distinct features of an event to build a new multi-featured representation requires additional processes that go beyond the processes involved in the storage of individual features themselves, and patients with schizophrenia have deficits in these processes. Such deficits might be characterized as a dysfunction of an episodic buffer system (e.g. Baddeley, 2000b) and/or may involve disruption of a frontal-hippocampal circuit that underlies feature binding (e.g. Mitchell et al., 2000a). It may also be that patients with schizophrenia have more difficulty than the control participants in evaluating multiple features at test (e.g. Mitchell et al., 2000b, Exp 2). Of course, these potential explanations are not mutually exclusive. Further studies are required to tease apart the relative contribution of a reduction in the number of features that can be maintained for several seconds, binding deficits, per se, and deficits in the ability to evaluate multiple features at test.

Finally, it would be fruitful to examine binding processes using both WM and long-term episodic tasks with the same participants and within the same study. This would shed additional light on the specificity vs. diversity of these processes across diverse aspects of human memory.

\section{References}

Aleman, A., Hijman, R., Dehaan, E.H.F., Kahn, R.S., 1999. Memory impairment in schizophrenia: a meta-analysis. American Journal of Psychiatry 156, 1358-1366.

American Psychiatric Association, 1994. Diagnostic and Statistical Manual of Mental Disorders, DSM-IV. 4 edn. American Psychiatric Association, Washington, DC.

Baddeley, A.D., 1986. Working Memory. Oxford University Press, Oxford.

Baddeley, A.D., 2000a. Short-term and working memory. In: Tulving, E., Craik, F.I.M. (Eds.), The Oxford Handbook of Memory. Oxford University Press, New York, pp. 77-92.

Baddeley, A.D., 2000b. The episodic buffer: a new component of working memory? Trends in Cognitive Sciences 4, 417-423.

Bentall, R.P., Baker, G.A., Havers, S., 1991. Reality monitoring and psychotic hallucinations. British Journal of Clinical Psychology 30, 213-222.
Chapman, L.J., Chapman, J.P., 1978. The measurement of differential deficit. Journal of Psychiatric Research 14, 303-311.

Cohen, J., Macwhinney, B., Flatt, M., Provost, J., 1993. Psyscope: an integrative graphic system for designing and controlling experiments in the psychology laboratory using Macintosh computers. Behavioral Research Methods. Instruments and Computers 25, 257-271.

Danion, J.-M., Rizzo, L., Bruant, A., 1999. Functional mechanisms underlying impaired recognition memory and conscious awareness in patients with schizophrenia. Archives of General Psychiatry 56, 639-644.

Glahn, D.C., Therman, S., Manninen, M., Huttunen, M., Kaprio, J., Lonnqvist, J., Cannon, T.D., 2003. Spatial working memory as an endophenotype for schizophrenia. Biological Psychiatry 53, 624-626.

Gold, J.M., Wilk, C.M., McMahon, R.P., Buchanan, R.W., Luck, S.J., 2003. Working memory for visual features and conjunctions in schizophrenia. Journal of Abnormal Psychology 112, 61-71.

Gras-Vincendon, A., Danion, J.-M., Grangé, D., Bilik, M., Willard-Schroeder, D., Sichel, J.-P., Singer, L., 1994. Explicit memory, repetition priming and cognitive skill learning in schizophrenia. Schizophrenia Research 13, 117-126.

Heinrichs, R.W., Zakzanis, K.K., 1998. Neurocognitive deficit in schizophrenia: a quantitative review of the evidence. Neuropsychology 12, 426-445.

Johnson, M.K., Chalfonte, B.L., 1994. Binding complex memories: the role of reactivation and the hippocampus. In: Schacter, D.L., Tulving, E. (Eds.), Memory Systems 1994. MIT Press, Cambridge, MA, pp. 311-350.

Keefe, R.S.E., 2000. Working memory dysfunction and its relevance to schizophrenia. In: Sharma, T., Harvey, P. (Eds.), Cognition in Schizophrenia. Oxford University Press, Oxford, pp. 16-50.

Luck, S.J., Vogel, E.K., 1997. The capacity of visual working memory for features and conjunctions. Nature 390, 279-281.

Mitchell, K.J., Johnson, M.K., Raye, C.L., D’Esposito, M., 2000a. fMRI evidence of age-related hippocampal dysfunction in feature binding in working memory. Cognitive Brain Research 10, 197-206.

Mitchell, K.J., Johnson, M.K., Raye, C.L., Mather, M., D’Esposito, M., 2000b. Aging and reflective processes of working memory: binding and test load deficits. Psychology and Aging 15, 527-541.

Prabhakaran, V., Narayanan, K., Zhao, Z., Gabrieli, J.D.E., 2000. Integration of diverse information in working memory within the frontal lobe. Nature Neuroscience 3, 85-90.

Rankin, P.M., O'Carroll, P.J., 1995. Reality discrimination, reality monitoring and disposition towards hallucination British Journal of Clinical Psychology 34, 517-528.

Rizzo, L., Danion, J.-M., Van der Linden, M., Grangé, D., Rohmer, J.G., 1996. Impairment of memory for spatial context in schizophrenia. Neuropsychology 10, 376-384.

Salamé, P., Danion, J.-M., Peretti, C.S., Cuervo, C., 1998. The state of functioning of working memory in schizophrenia. Schizophrenia Research 30, 11-29. 
Schwartz, B.L., Deutsch, L.H., Cohen, C., Warden, D., Deutsch, S.I., 1991. Memory for temporal order in schizophrenia. Biological Psychiatry 29, 329-339.

Snodgrass, J.G., Vanderwart, M., 1980. A standardized set of 260 pictures: norms for name agreement, image agreement, familiarity, and visual complexity. Journal of Experimental Psychology: Human Learning 6, 174-215.
Wechsler, D., 1981. Manual for the Wechsler Adult Intelligence Scale. Psychological Corporation, New York.

Wheeler, M.E., Treisman, A.M., 2002. Binding in short-term visual memory. Journal of Experimental Psychology: General 131, 48-64. 